\title{
Image of Dynamic Local Exchange Interactions in the dc Magnetoresistance of Spin-Polarized Current through a Dopant
}

\author{
Stephen R. McMillan, ${ }^{1,2}$ Nicholas J. Harmon $\odot,{ }^{1, *}$ and Michael E. Flatté ${ }^{1,2,3}$ \\ ${ }^{1}$ Optical Science and Technology Center, and Department of Physics and Astronomy, University of Iowa, Iowa City, Iowa 52242, USA \\ ${ }^{2}$ Pritzker School of Molecular Engineering, University of Chicago, Chicago, Illinois 60637, USA \\ ${ }^{3}$ Department of Applied Physics, Eindhoven University of Technology, P.O. Box 513, 5600 MB Eindhoven, Netherlands
}

(Received 11 July 2019; revised 15 September 2020; accepted 10 November 2020; published 17 December 2020)

\begin{abstract}
We predict strong, dynamical effects in the dc magnetoresistance of current flowing from a spinpolarized electrical contact through a magnetic dopant in a nonmagnetic host. Using the stochastic Liouville formalism we calculate clearly defined resonances in the dc magnetoresistance when the applied magnetic field matches the exchange interaction with a nearby spin. At these resonances spin precession in the applied magnetic field is canceled by spin evolution in the exchange field, preserving a dynamic bottleneck for spin transport through the dopant. Similar features emerge when the dopant spin is coupled to nearby nuclei through the hyperfine interaction. These features provide a precise means of measuring exchange or hyperfine couplings between localized spins near a surface using spin-polarized scanning tunneling microscopy, without any ac electric or magnetic fields, even when the exchange or hyperfine energy is orders of magnitude smaller than the thermal energy.
\end{abstract}

DOI: 10.1103/PhysRevLett.125.257203

Localized spin states with long spin coherence times are a fundamental element of the quantum coherent spin systems [1,2] that underlie quantum sensing [3] and quantum information processing [4]. Dopants, and some other controlled defects, in semiconductor or insulator hosts provide a robust realization of these localized coherent spins [5,6]. Optical probes have revealed the spin dynamics of such spin centers, along with their coherent interactions with neighboring spins [7-12]. Similar evidence of all-electrical probing of coherent interactions has been achieved at low temperatures in dopants in large magnetic fields [13,14], and with electron spin resonance combined with spin-polarized scanning tunneling microscopy (STM-ESR) [15-18]. Low-field magnetoresistance without rf fields in much higher temperature dc electrical measurements, however, has been found in transport from a magnetic contact $[19,20]$ through traps to a nonmagnetic region, as well as in transport through traps in semiconductor devices [21]. These effects originate from coherent spin dynamics in resonant [22] or nonresonant (incoherent hopping) transport [20], and manifest as zerofield peaks or dips in the magnetoresistance unless the contact's spin polarization is time dependent [23].

Published by the American Physical Society under the terms of the Creative Commons Attribution 4.0 International license. Further distribution of this work must maintain attribution to the author(s) and the published article's title, journal citation, and DOI.
Here we predict that the $\mathrm{dc}$ magnetoresistance of electrical current through the spin state of a single dopant, which can be measured, e.g., using spin-polarized STM (SP-STM [24-26]), should directly image the coherent effects of the environment on the dopant's spin dynamics, e.g., exchange or hyperfine interactions, even at temperatures far larger than the energy scales of those interactions. These sharp features in the dc magnetoresistance do not require spin-orbit interaction in the system, and will persist even when the charge transport process is incoherent. The single dopant would reside in the surface region of a weakly conducting nonmagnetic host, and experience the effect of a second nuclear or electron spin through isotropic exchange or hyperfine interactions. The charge and spin magnitude of the second spin is fixed, such as for the electronic spin of a deep trap or a nuclear spin. Strong dc magnetoresistance features are seen when the dopant's spin precession due to the applied magnetic field cancels the precession due to the exchange or hyperfine interaction with the second spin. These features can be resolved at very small magnetic fields and thus should permit the measurement of mT hyperfine interactions and $\mu \mathrm{eV}$ exchange interaction strengths, such as are predicted for spin centers in wide-gap semiconductors such as diamond [27].

Double quantum dot measurements of spin bottlenecks [28] would not see these features, as the spin polarization transverse to the applied magnetic field must be measured. These features appear in the dc current, not in the noise as some other spin-dynamical effects manifest [29]. The coherent features we predict in the magnetoresistance will remain detectable, even when the thermal energy exceeds 
the energy scales of spin interactions with the environment by orders of magnitude, if the nanosecond transport times of the junction are much shorter than the dopant's spin coherence time (e.g., $100 \mathrm{~ns}$ for $\mathrm{Mn}$ in GaAs [30] and $200 \mu \mathrm{s}$ for $\mathrm{P}$ in $\mathrm{Si}[6])$. This differs from SP-STM measurements of spin excitations during inelastic tunneling spectroscopy [31,32], which cannot be resolved when the temperature exceeds the energy of the spin excitation. More generally, these coherent features may emerge in any flow of current through localized states in nanoscale devices containing contacts with spin polarization oblique to an external field.

The detailed configuration is shown in Fig. 1. Here we focus on a specific situation in which the dopant through which transport occurs (transport site, green) possesses two states, one of which is spin-0 and "empty," and the other is spin- $1 / 2$ and "full," corresponding to an additional electron. When full the transport site interacts with a second spin-1/2 (spectator site, red), whose charge state is unaffected during transport. The details of the magnetoresistive curves, and the visibility of coherent features within them, depend on the relative strength of the transport-spin-spectator-spin interaction $J$ and the hopping rates, here $\gamma_{\text {on }}$ from the nonmagnetic host to the transport

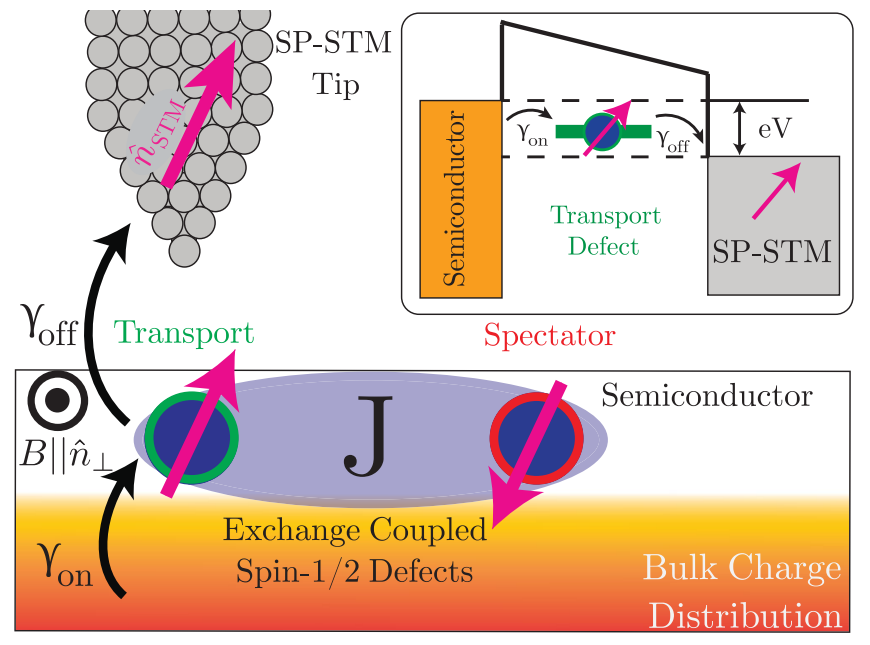

FIG. 1. Schematic current path for an electron through a single dopant (transport site, in green) that is exchange coupled to another spin-1/2 (spectator site, in red). The transport site has two charge states, empty (spin-0) and full (spin-1/2), and the spectator site's charge state is stable. These spins reside near the surface of the nonmagnetic host and the Coulomb repulsion at the transport site is assumed to be sufficiently large to prevent double occupation (see inset). Hopping from the transport site to a spinpolarized scanning tunneling microscope tip occurs with rate $\gamma_{\text {off }}$, which is controllable by moving the STM tip relative to the surface. Replenishment of the occupation of the transport site from the nonmagnetic host occurs with a rate $\gamma_{\text {on }}$. If the host is a semiconductor the charge distribution can be adjusted by the STM voltage $V$ through tip induced band bending, which adjusts $\gamma_{\text {on }}$ and $\gamma_{\text {off }}$. site, and $\gamma_{\text {off }}$ from the transport site to the SP-STM tip; similar features are expected for a wide variety of transport and spectator spin states. The use of the STM provides atomic-scale resolution and permits direct selection of the dopant spin to be probed, and it also provides direct means to independently tune $\gamma_{\text {on }}$ and $\gamma_{\text {off }}$. By moving the tip towards or away from the surface the $\gamma_{\text {off }}$ can be adjusted [33-35], and through tip-induced band bending both $\gamma_{\text {off }}$ and $\gamma_{\text {on }}$ can be modified [36,37]. We find the most sensitive configurations correspond to $\gamma_{\text {off }} \ll \gamma_{\text {on }}$, which is the expected experimental situation under ordinary conditions. We consider the SP-STM to be polarized along the axis $\hat{n}_{\text {STM }}$, which can point in any direction (Fig. 1).

Our main results are the predicted finite-field dips (resonances) in the dc magnetoresistance in Fig. 2 that occur due to degeneracies in the transport states and the formation of bottlenecks. Coupling dopants to spin-polarized contacts has been shown to lead to zero-field current bottlenecks resulting from dopant polarization antiparallel to the magnetization of the contact [20]. The application of an external magnetic field, $\mathbf{B}=B \hat{n}_{\perp}$, along an axis $\hat{n}_{\perp}$ perpendicular to the contact spin polarization $\left(\hat{n}_{\mathrm{STM}} \cdot \hat{n}_{\perp}=0\right)$, releases the bottleneck by precessing the spin and produces a characteristic dip feature in the zero-field dc magnetoresistance $[19,20]$. Figure 2, however, shows two additional features at finite field when the Zeeman energy of the transport spin equals its exchange energy with the spectator spin. New bottlenecks emerge under these conditions, because the triplet state polarized parallel or antiparallel to the external field (depending on the sign of $J$ ) becomes degenerate with the singlet state $(S)$ of these two spins at this external field. We find this dip in

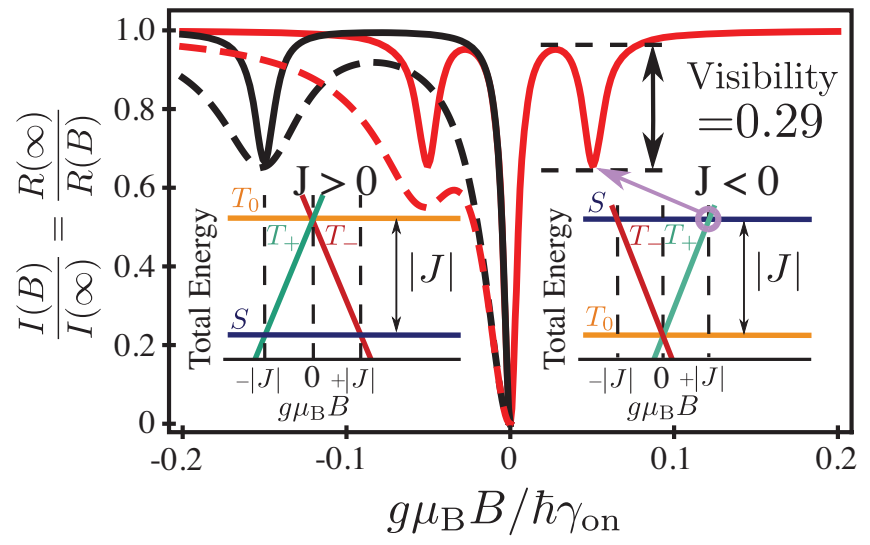

FIG. 2. Magnetoresistance of current through spin-1/2 transport dopant for exchange splitting of $J=0.05 \hbar \gamma_{\text {on }}$ (red) and $J=0.15 \hbar \gamma_{\text {on }}$ (black). The dips at finite field result from resonances between the triplet and singlet states and are independent of the sign of $J$ (insets, with the triplet states in the $\hat{n}_{\perp}$ basis). These features depend on the transport rates, broadening as $\gamma_{\text {off }}$ is increased from $\gamma_{\text {on }} / 100$ (solid) to $\gamma_{\text {on }} / 25$ (dashed). The visibility (the difference between the low-field maxima and finitefield minima) decreases as $\gamma_{\text {off }}$ increases. 
current has a maximal value of $1 / 3$ of the $B=0 \mathrm{dip}$. The dip's visibility (the difference between the low-field maximum and finite-field minimum, as indicated in Fig. 2) decreases as $\gamma_{\text {off }}$ increases. Although our description here is for a $100 \%$ spin-polarized SP-STM tip, these features will occur for any finite spin polarization, with a reduced amplitude (as seen for zero-field dips in previous work [20]).

The density matrix requires time evolution terms describing coherent evolution of the spins, originating from the magnetic field and exchange, and incoherent processes that inject or extract carriers from the transport site (thus producing current). We use the stochastic Liouville formalism $[38,39]$, which has successfully predicted the manipulation of electroluminescence from organic LEDs [40], exciplex light emission [41], and nuclear spin influences on electron transport [42]. The Hamiltonian describing coherent evolution of the spins at both the spectator and transport sites is

$$
\hat{H}_{s t}=g \mu_{\mathrm{B}} \mathbf{B} \cdot\left(\mathbf{S}_{s}+\mathbf{S}_{t}\right)+J \mathbf{S}_{s} \cdot \mathbf{S}_{t}
$$

where $\mathbf{S}_{s(t)}$ represents the spin operator at the spectator (transport) site, $g$ is the Landé $g$ factor, and $\mu_{B}$ is the Bohr magneton. As the spin-spin interaction is very general in Eq. (1), this description also applies to dipolar and hyperfine interactions with a spectator spin. When the transport site is unoccupied, the Hamiltonian becomes

$$
\hat{H}_{s}=g \mu_{\mathrm{B}} \mathbf{B} \cdot \mathbf{S}_{s} .
$$

The coherent evolution of the density operator is

$$
\left(\frac{\partial \rho_{i}(t)}{\partial t}\right)_{\mathrm{coh}}=-\frac{i}{\hbar}\left[\hat{H}_{i}, \rho_{i}(t)\right]
$$

where the subscript $i$ is used to denote either the operator for the isolated spectator $(i=s)$ or the combined spectator and transport spin $(i=s t)$ and [,] represents the commutator.

Transport occurs when the dopant at the transport site couples to unpolarized bulk states and the SP-STM, resulting in the generation and extraction of carriers. These processes will not preserve the trace of the individual subspaces of the system, but will preserve the total trace, $\operatorname{Tr} \rho_{s}(t)+\operatorname{Tr} \rho_{s t}(t)=1$, and decohere off-diagonal elements of $\rho_{s t}$. The polarization of the SP-STM tip can be written as an operator $\hat{M}_{\text {off }}\left(\hat{n}_{\text {STM }}\right)$, where $\hat{n}_{\text {STM }}$ is the orientation of the SP-STM spin polarization. In general, for a single spin-1/2 manifold [43] the interaction operator can be written as a linear superposition of spin-polarized projection operators parallel $\hat{\pi}\left(\hat{n}_{\text {STM }}\right)$ and antiparallel $\hat{\pi}\left(-\hat{n}_{\mathrm{STM}}\right)$ to $\hat{n}_{\mathrm{STM}}$,

$$
\begin{aligned}
\hat{m}\left(\hat{n}_{\mathrm{STM}}\right) & =a \hat{\pi}\left(\hat{n}_{\mathrm{STM}}\right)+b \hat{\pi}\left(-\hat{n}_{\mathrm{STM}}\right) \\
& =\frac{1}{2}\left\{(a+b) \hat{\sigma}_{0}+(a-b) \hat{n}_{\mathrm{STM}} \cdot \hat{\sigma}\right\},
\end{aligned}
$$

where $a$ and $b$ are spin parallel and antiparallel interaction amplitudes, and $a+b=1$. In the case of a manifold describing two spin- $1 / 2$ dopants, the interaction operator results from the direct product of two single spin operators,

$$
\hat{M}\left(\hat{n}_{\mathrm{STM}}\right)=\hat{m}_{t}\left(\hat{n}_{\mathrm{STM}}\right) \otimes \hat{m}_{s}\left(\hat{n}_{\mathrm{STM}}\right),
$$

where $\quad \hat{m}_{t}\left(\hat{n}_{\mathrm{STM}}\right)=a_{t} \hat{\pi}\left(\hat{n}_{\mathrm{STM}}\right)+b_{t} \hat{\pi}\left(-\hat{n}_{\mathrm{STM}}\right) \quad$ and $\hat{m}_{s}\left(\hat{n}_{\mathrm{STM}}\right)=a_{s} \hat{\pi}\left(\hat{n}_{\mathrm{STM}}\right)+b_{s} \hat{\pi}\left(-\hat{n}_{\mathrm{STM}}\right)$. For a $100 \%$ spinpolarized STM tip, the coefficients for $\hat{M}_{\text {off }}$ are $a_{t}^{\text {off }}=1, b_{t}^{\text {off }}=0, a_{s}^{\text {off }}=b_{s}^{\text {off }}=1 / 2 . \hat{M}_{\text {on }}$ couples the bulk to the dopant, and for unpolarized bulk states has the coefficients $a_{t}^{\text {on }}=a_{s}^{\text {on }}=b_{t}^{\text {on }}=b_{s}^{\text {on }}=1 / 2$. We assume a large on-site Coulomb repulsion that excludes double occupation of the transport site. The spin state of the carrier entering the transport site from the bulk is determined by the spin polarization of the bulk $\left(\hat{M}_{\text {on }}\right)$ and the spin state of the spectator is determined by the density matrix for the $2 \times 2$ manifold, $\rho_{s}(t)$. Thus the current operator for reoccupation of the transport site,

$$
\hat{i}_{\text {on }}=\frac{e \gamma_{\text {on }}}{2} \hat{m}_{t}^{\text {on }}\left(\hat{n}_{\mathrm{STM}}\right) \otimes \rho_{s}(t),
$$

where $e$ is the electronic charge.

The current from the dopant to the SP-STM depends on the availability of spin states in the tip $\left(\hat{M}_{\text {off }}\right)$ and the twodopant density matrix $\rho_{s t}$. The current operator for dopantto-tip transport is

$$
\hat{i}_{\text {off }}=\frac{e \gamma_{\text {off }}}{2}\left\{\hat{M}_{\text {off }}, \rho_{s t}(t)\right\}
$$

where the anticommutator $\{$,$\} guarantees the operator is$ Hermitian and properly decoheres off-diagonal elements of $\rho_{s t}$ [44]. The current onto and off of the transport site is found from the trace of the current operators. The total current onto and off of the transport site must be equal: $\operatorname{Tr} \hat{i}_{\text {on }}=\operatorname{Tr} \hat{i}_{\text {off }}=I$, however, the spin current $I_{\text {on(off })}^{\text {spin }}=$ $\operatorname{Tr}\left[\hat{i}_{\text {on(off) }} \boldsymbol{\sigma}\right]$ is not conserved due to decoherence.

The appropriate term can now be added to the stochastic Liouville equation for carrier generation

$$
\left(\frac{\partial \rho_{s t}(t)}{\partial t}\right)_{\text {gen }}=\gamma_{\text {on }} \hat{m}_{t}^{\text {on }}\left(\hat{n}_{\mathrm{STM}}\right) \otimes \rho_{s}(t)
$$

and extraction

$$
\left(\frac{\partial \rho_{s t}(t)}{\partial t}\right)_{\mathrm{ext}}=-\gamma_{\mathrm{off}}\left\{\hat{M}_{\mathrm{off}}, \rho_{s t}(t)\right\}
$$


The total equation for the $4 \times 4$ density operator $\rho_{s t}$ is then

$$
\begin{aligned}
\frac{\partial \rho_{s t}(t)}{\partial t}= & -\frac{i}{\hbar}\left[\hat{H}_{s t}, \rho_{s t}(t)\right]+\gamma_{\mathrm{on}} \mathbb{1}_{t} \otimes \rho_{s}(t) \\
& -2 \gamma_{\mathrm{off}}\left\{\hat{M}_{\mathrm{off}}, \rho_{s t}(t)\right\},
\end{aligned}
$$

where $\mathbb{1}_{t}$ is the identity matrix in the transport subspace and the relation $2 \hat{m}_{t}^{\text {on }}\left(\hat{n}_{\text {STM }}\right)=\mathbb{1}_{t}$ has been used. Equation (10) is coupled to the single spin density operator $\rho_{s}$ through the generation term. The trace of the total system is preserved and thus $\operatorname{Tr} \rho_{s}+\operatorname{Tr} \rho_{s t}=1$; generation (decay) of the $4 \times 4$ density matrix must be balanced by the decay (generation) of the $2 \times 2$ density matrix allowing one to derive for the isolated spectator,

$$
\begin{aligned}
\frac{\partial \rho_{s}(t)}{\partial t}= & -\frac{i}{\hbar}\left[\hat{H}_{s}, \rho_{s}(t)\right]+2 \gamma_{\mathrm{off}} \operatorname{Tr}_{t}\left\{\hat{M}_{\mathrm{off}}, \rho_{s t}(t)\right\} \\
& -2 \gamma_{\mathrm{on}} \rho_{s}(t)
\end{aligned}
$$

where $\operatorname{Tr}_{t}$ is a partial trace over the transport subspace.

Setting $\partial \rho / \partial t=0$ and tracing the current operators [Eqs. (6) and (7)] yields an analytic (but cumbersome) solution for the steady-state current (see Supplemental Material [45], along with additional derivations). The expressions for the near zero field feature agree with calculations of current through mediating dopants in insulating spacers, during either resonant [22] or incoherent [20] transport. However, we are interested in the expressions when the Zeeman energy $\left(|\tilde{B}|\right.$, where $\left.\tilde{B}=g \mu_{B} B\right)$, approaches the exchange energy $J$. An approximate expression valid for $\gamma_{\text {on }} \gg|\tilde{B}| / \hbar,|J| / \hbar \gg \gamma_{\text {off }}$ near the $\tilde{B}= \pm J$ resonance is

$$
I=\left(\frac{\gamma_{\text {off }}}{2}\right) \frac{6 J^{2} \hbar^{2} \gamma_{\text {off }}^{2}+4\left(\tilde{B}^{2}-J^{2}\right)^{2}}{9 J^{2} \hbar^{2} \gamma_{\text {off }}^{2}+4\left(\tilde{B}^{2}-J^{2}\right)^{2}}
$$

where the prefactor is the limiting value away from resonant features. Thus the relative current has a limiting value for $|\tilde{B}|=|J|$ of $2 / 3$, corresponding to a visibility of $1 / 3$ as seen below in numerical calculations. Figure 2 shows the calculated magnetoresistance, for a $100 \%$ spinpolarized STM tip.

Figure 3 shows the visibility for different $\gamma_{\text {off }} / \gamma_{\text {on }}$ as a function of $|J| / \hbar \gamma_{\text {on }}$. At a finite-field dip in current one of the triplet states ( $T_{+}$or $T_{-}$along $\hat{n}_{\perp}$, depending on $J$ 's sign) becomes degenerate with the singlet state $S$. The other two triplet states do not participate in the bottleneck. The current bottleneck manifests as a reduced rate, for one of the two degenerate states, for the transport site's electron to hop to the SP-STM tip. The linear combination of those two states that has the lowest transport-site tunneling probability to the SP-STM tip is $\left|\phi_{b}\right\rangle$, and the orthogonal state is $\left|\bar{\phi}_{b}\right\rangle$, where

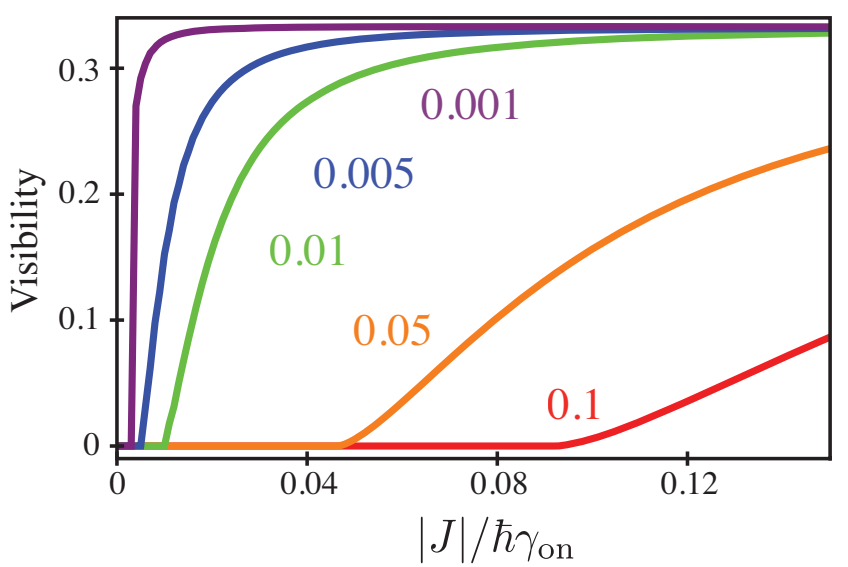

FIG. 3. Visibility of the finite-field feature (resonance) as a function of the exchange parameter plotted for different values of $\gamma_{\text {off }} / \gamma_{\text {on }}$. Smaller ratios yield higher visibility due to extended spin evolution in the exchange field.

$\left|\phi_{b}\right\rangle=6^{-1 / 2}|\uparrow \Uparrow\rangle+6^{-1 / 2}|\downarrow \Downarrow\rangle+(2 / 3)^{1 / 2}|\downarrow \Uparrow\rangle$,

$\left|\bar{\phi}_{b}\right\rangle=(12)^{-1 / 2}(|\uparrow \Uparrow\rangle+|\downarrow \Downarrow\rangle-|\downarrow \uparrow\rangle)+(3 / 4)^{1 / 2}|\uparrow \Downarrow\rangle$.

The first spin (single arrow) represents the transport site and the second (double arrow) represents the spectator site, and the axes are along the SP-STM spin polarization. $\phi_{b}$ has a transport spin polarization of $2 / 3$, which produces a maximum dip in the current of $1 / 3$ as derived in the Supplemental Material [45], as plotted in Figs. 2 and 3, and as apparent in Eq. (12) for $|\tilde{B}|=|J|$.

Figure 3 also illustrates the increase in resolution and precision gained by minimizing the hopping ratio. For a dopant-to-tip hopping rate of $\gamma_{\text {off }}=1 \mathrm{~ns}^{-1}$ we predict exchange energy resolution $\sim \mu \mathrm{eV}$. If the energy splittings to double occupancy of the transport site greatly exceed the thermal energy, and the spin coherence times of the spins exceed the timescales set by the smallest energies in the problem (Zeeman energy, exchange energy, or hopping) then the magnetoresistance features survive; observations of zero-field dips are seen at room temperature [21]. The energy sensitivity surpasses the practical limits of STM spectroscopic probes of exchange interactions in semiconductor dopant pairs $[46,47]$, and could be measured at temperatures far above where inelastic spin excitations can be imaged for adatoms [31,32].

Figure 4(a) shows calculations for the degree of spin polarization as a function of the hopping ratio for different values of the junction parameters. The dashed lines represent the spectator spin and the solid lines represent the transport spin. In Figs. 4(b)-4(d) the occupation probability for each state in the product basis as a function of $B$ is shown. An analytic treatment for $|\tilde{B}|=|J|$ and the limit $\gamma_{\text {off }} \ll J / \hbar \ll \gamma_{\text {on }}$ [similar to panel (d)], described in the Supplemental Material [45], predicts a polarization for 

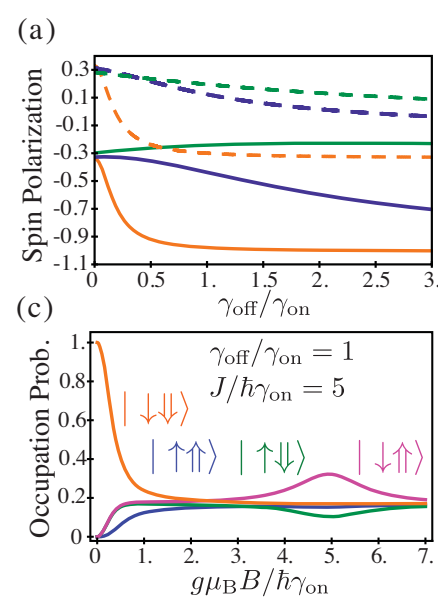

(b)

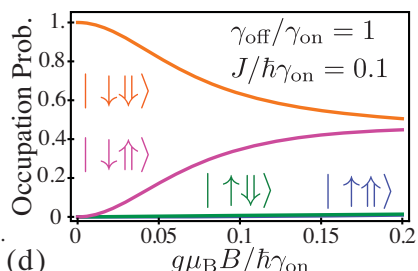

(d)

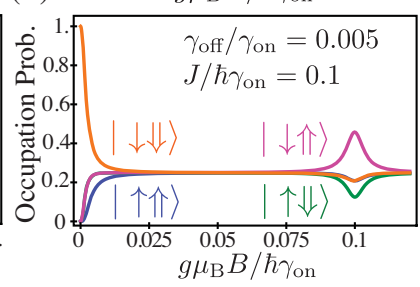

FIG. 4. (a) Spin polarization at resonance $(\tilde{B}=J)$ for $J>0$ as a function of hopping ratio plotted for different values of the exchange coupling: $J / \hbar \gamma_{\text {on }}=0.1$ (orange), $J / \hbar \gamma_{\text {on }}=1$ (blue), $J / \hbar \gamma_{\text {on }}=5$ (green). The dashed lines represent the spectator spin and solid lines represent the transport spin. (b)-(d) Occupation probability for the product states in the $\hat{n}_{\mathrm{STM}}$ basis as a function of the transverse magnetic field. Shown are (b) $\gamma_{\text {off }} / \gamma_{\text {on }}=1$, $\gamma_{\text {off }} \gg J / \hbar$, (c) $\gamma_{\text {off }} / \gamma_{\text {on }}=1, \gamma_{\text {off }} \ll J / \hbar$, and (d) $\gamma_{\text {on }} \gg J / \hbar \gg$ $\gamma_{\text {off }}$. Good visibility is realized when $\gamma_{\text {off }} \ll J / \hbar$, and especially for (d).

$|\downarrow \uparrow\rangle$ of $11 / 24$, for $|\uparrow \Downarrow\rangle$ of $1 / 8$, and for $|\uparrow \uparrow\rangle$ and $|\downarrow \Downarrow\rangle$ of $5 / 24$, which are all different from the random average of $1 / 4$. These results are very similar to those shown in Fig. 4(d). The spectator spin is also dynamically polarized during this process, even when the transport spin state is empty; we find for the limiting conditions above, similar to Fig. 4(d) a polarization of $1 / 2$ antiparallel to the magnetic field direction and 1/4 parallel to the SP-STM polarization direction for a total polarization of $\sqrt{5} / 4$.

This material is based on work supported by the U.S. Department of Energy, Office of Science, Office of Basic Energy Sciences, under Award No. DE-SC0016447 and its renewal DE-SC0016379.

*Present address: Department of Physics, University of Evansville, Evansville, Indiana 47722, USA.

[1] Semiconductor Spintronics and Quantum Computation, edited by D. D. Awschalom, N. Samarth, and D. Loss (Springer Verlag, Heidelberg, 2002), https://doi.org/10 .1007/978-3-662-05003-3.

[2] R. Hanson, L. P. Kouwenhoven, J. R. Petta, S. Tarucha, and L. M. K. Vandersypen, Spins in few-electron quantum dots, Rev. Mod. Phys. 79, 1217 (2007).

[3] C. L. Degen, F. Reinhard, and P. Cappellaro, Quantum sensing, Rev. Mod. Phys. 89, 035002 (2017).

[4] M. Nielsen and I. Chuang, Quantum Computation and Quantum Information (Cambridge University Press, New York, 2010), https://doi.org/10.1017/CBO978 0511976667.

[5] P. M. Koenraad and M. E. Flatté, Single dopants in semiconductors, Nat. Mater. 10, 91 (2011).

[6] J. J. Pla, K. Y. Tan, J. P. Dehollain, W. H. Lim, J. J. L. Morton, D. N. Jamieson, A. S. Dzurak, and A. Morello, A single-atom electron spin qubit in silicon, Nature (London) 489, 541 (2012).

[7] G. Balasubramanian, P. Neumann, D. Twitchen, M. Markham, R. Kolesov, N. Mizuochi, J. Isoya, J. Achard, J. Beck, J. Tissler, V. Jacques, P. R Hemmer, F. Jelezko, and J. Wrachtrup, Ultralong spin coherence time in isotopically engineered diamond, Nat. Mater. 8, 383 (2009).

[8] K. Ohno, F. J. Heremans, L. C. Bassett, B. A. Myers, D. M. Toyli, A. C. Bleszynski Jayich, C. J. Palmstrøm, and D. D. Awschalom, Engineering shallow spins in diamond with nitrogen delta-doping, Appl. Phys. Lett. 101, 082413 (2012).

[9] V. V. Dobrovitski, G. D. Fuchs, A. L. Falk, C. Santori, and D. D. Awschalom, Quantum control over single spins in diamond, Annu. Rev. Condens. Matter Phys. 4, 23 (2013).

[10] B. A. Myers, A. Das, M. C. Dartiailh, K. Ohno, D. D. Awschalom, and A. C. Bleszynski Jayich, Probing Surface Noise with Depth-Calibrated Spins in Diamond, Phys. Rev. Lett. 113, 027602 (2014).

[11] D. J. Christle, P. V. Klimov, C. F. de las Casas, K. Szász, V. Ivády, V. Jokubavicius, J. Ul Hassan, M. Syväjärvi, W. F. Koehl, T. Ohshima, N. T. Son, E. Janzén, Á. Gali, and D. D. Awschalom, Isolated Spin Qubits in SiC with a HighFidelity Infrared Spin-to-Photon Interface, Phys. Rev. X 7, 021046 (2017).

[12] R. Nagy, M. Niethammer, M. Widmann, Y. Chen, P. Udvarhelyi, C. Bonato, J. Ul Hassan, R. Karhu, I. G. Ivanov, N. T. Son, J. R. Maze, T. Ohshima, Ö. O. Soykal, Á. Gali, S. Lee, F. Kaiser, and J. Wrachtrup, High-fidelity spin and optical control of single silicon-vacancy centres in silicon carbide, Nat. Commun. 10, 1954 (2019).

[13] M. A. Broome, S. K. Gorman, M. G. House, S. J. Hile, J. G. Keizer, D. Keith, C. D. Hill, T. F. Watson, W. J. Baker, L. C. L. Hollenberg, and M. Y. Simmons, Two-electron spin correlations in precision placed donors in silicon, Nat. Commun. 9, 980 (2018).

[14] P. Pakkiam, A. V. Timofeev, M. G. House, M. R. Hogg, T. Kobayashi, M. Koch, S. Rogge, and M. Y. Simmons, Single-Shot Single-Gate rf Spin Readout in Silicon, Phys. Rev. X 8, 041032 (2018).

[15] S. Baumann, W. Paul, T. Choi, C. P. Lutz, A. Ardavan, and A. J. Heinrich, Electron paramagnetic resonance of individual atoms on a surface, Science 350, 417 (2015).

[16] Y. Bae, K. Yang, P. Willke, T. Choi, A. J. Heinrich, and C. P. Lutz, Enhanced quantum coherence in exchange coupled spins via singlet-triplet transitions, Sci. Adv. 4, eaau4159 (2018).

[17] P. Willke, Y. Bae, K. Yang, J. L. Lado, A. Ferrón, T. Choi, A. Ardavan, J. Fernández-Rossier, A. J. Heinrich, and C. P. Lutz, Hyperfine interaction of individual atoms on a surface, Science 362, 336 (2018).

[18] K. Yang, P. Willke, Y. Bae, A. Ferrón, J. L. Lado, A. Ardavan, J. Fernández-Rossier, A. J. Heinrich, and C. P. Lutz, Electrically controlled nuclear polarization of individual atoms, Nat. Nanotechnol. 13, 1120 (2018).

[19] O. Txoperena, Y. Song, L. Qing, M. Gobbi, L. E. Hueso, H. Dery, and F. Casanova, Impurity-Assisted Tunneling 
Magnetoresistance Under a Weak Magnetic Field, Phys. Rev. Lett. 113, 146601 (2014).

[20] H. Inoue, A. G. Swartz, N. J. Harmon, T. Tachikawa, Y. Hikita, M. E. Flatté, and H. Y. Hwang, Origin of the Magnetoresistance in Oxide Tunnel Junctions Determined through Electric Polarization Control of the Interface, Phys. Rev. X 5, 041023 (2015).

[21] J. P. Ashton, S. J. Moxim, P. M. Lenahan, C. G. McKay, R. J. Waskiewicz, K. J. Myers, M. E. Flatté, N. J. Harmon, and C. D. Young, A new analytical tool for the study of radiation effects in 3-D integrated circuits: Near-zero field magnetoresistance spectroscopy, IEEE Trans. Nucl. Sci. 66, 428 (2019).

[22] Y. Song and H. Dery, Magnetic-Field-Modulated Resonant Tunneling in Ferromagnetic-Insulator-Nonmagnetic Junctions, Phys. Rev. Lett. 113, 047205 (2014).

[23] N. J. Harmon and M. E. Flatté, Theory of spin-coherent electrical transport through a defect spin state in a metal/ insulator/ferromagnet tunnel junction undergoing ferromagnetic resonance, Phys. Rev. B 98, 035412 (2018).

[24] D. Wortmann, S. Heinze, Ph. Kurz, G. Bihlmayer, and S. Blügel, Resolving Complex Atomic-Scale Spin Structures by Spin-Polarized Scanning Tunneling Microscopy, Phys. Rev. Lett. 86, 4132 (2001).

[25] R. Wiesendanger, Mapping spin structures on the atomic scale, Europhysics News 38, 16 (2007).

[26] S. Loth, C. P. Lutz, and A. J. Heinrich, Spin-polarized spin excitation spectroscopy, New J. Phys. 12, 125021 (2010).

[27] V. R. Kortan, C. Şahin, and M. E. Flatté, Nanometer-scale exchange interactions between spin centers in diamond, Phys. Rev. B 93, 220402(R) (2016).

[28] J. R. Petta, A. C. Johnson, J. M. Taylor, E. A. Laird, A. Yacoby, M. D. Lukin, C. M. Marcus, M. P. Hanson, and A. C. Gossard, Coherent manipulation of coupled electron spins in semiconductor quantum dots, Science 309, 2180 (2005).

[29] A. Burtzlaff, A. Weismann, M. Brandbyge, and R. Berndt, Shot Noise as a Probe of Spin-Polarized Transport Through Single Atoms, Phys. Rev. Lett. 114, 016602 (2015).

[30] R. C. Myers, M. H. Mikkelsen, J.-M. Tang, A. C. Gossard, M. E. Flatté, and D. D. Awschalom, Zero-field optical manipulation of magnetic ions in semiconductors, Nat. Mater. 7, 203 (2008).

[31] C. F. Hirjibehedin, C. P. Lutz, and A. J. Heinrich, Spin coupling in engineered atomic structures, Science 312, 1021 (2006).

[32] R. Wiesendanger, Spin mapping at the nanoscale and atomic scale, Rev. Mod. Phys. 81, 1495 (2009).
[33] Z. Zhang and J. T. Yates, Jr., Band bending in semiconductors: Chemical and physical consequences at surfaces and interfaces, Chem. Rev. 112, 5520 (2012).

[34] R. T. Tung, Recent advances in Schottky barrier concepts, Mater. Sci. Eng. R 35, 1 (2001).

[35] D. H. Lee and J. A. Gupta, Tunable field control over the binding energy of single dopants by a charged vacancy in GaAs, Science 330, 1807 (2010).

[36] G. J. de Raad, D. M. Bruls, P. M. Koenraad, and J. H. Wolter, Interplay between tip-induced band bending and voltage-dependent surface corrugation on GaAs(110) surfaces, Phys. Rev. B 66, 195306 (2002).

[37] R. M. Feenstra, J. A. Stroscio, J. Tersoff, and A. P. Fein, Atom-Selective Imaging of the GaAs(110) Surface, Phys. Rev. Lett. 58, 1192 (1987).

[38] R. Kubo, Stochastic Liouville equations, J. Math. Phys. (N.Y.) 4, 174 (1963).

[39] N. G. Van Kampen, Stochastic Processes in Physics and Chemistry, Vol. 1 (Elsevier, New York, 1992), https://doi .org/10.1016/B978-0-444-52965-7.X5000-4.

[40] N. J. Harmon, M. Wohlgenannt, and M. E. Flatté, Manipulation of the electroluminescence of organic light-emitting diodes via fringe fields from patterned magnetic domains, Appl. Phys. Lett. 109, 243303 (2016).

[41] Y. Wang, K. Sahin-Tiras, N. J. Harmon, M. Wohlgenannt, and M. E. Flatté, Immense Magnetic Response of Exciplex Light Emission Due to Correlated Spin-Charge Dynamics, Phys. Rev. X 6, 011011 (2016).

[42] S. K. Gorman, M. A. Broome, W. J. Baker, and M. Y. Simmons, Impact of nuclear spin dynamics on electron transport through donors, Phys. Rev. B 92, 125413 (2015).

[43] P. S. Farago, Electron spin polarization, Rep. Prog. Phys. 34, 1055 (1971).

[44] R. Haberkorn, Density matrix description of spin-selective radical pair reactions, Mol. Phys. 32, 1491 (1976).

[45] See Supplemental Material at http://link.aps.org/ supplemental/10.1103/PhysRevLett.125.257203 for the full general analytic expression for the current, limiting expressions for the current near the zero-field and finite-field resonances, analytic expressions for the various spin polarizations, a derivation of the bottleneck state wave function, and an approximate analytic model that provides further heuristic insight.

[46] M.E. Flatté and D.E. Reynolds, Local spectrum of a superconductor as a probe of interactions between magnetic impurities, Phys. Rev. B 61, 14810 (2000).

[47] D. Kitchen, A. Richardella, J.-M. Tang, M. E. Flatté, and A. Yazdani, Atom-by-atom substitution of $\mathrm{Mn}$ in GaAs and visualization of their hole-mediated interactions, Nature (London) 442, 436 (2006). 